\title{
EDITORIAL
}

\section{BREAK THE PANDEMIC CIRCUIT WITH PROFESSIONAL VALUES AND COMMITMENT}

\author{
Sally Wai-Chi Chan \\ President and Professor \\ Tung Wah College, Hong Kong, China
}

Having tormented the world for over 2 years, the COVID pandemic posts a new risk to human. The new variant, called Omicron, is very potent and more transmissible. Healthcare services all over the world are facing a much more difficult situation. So is the nursing profession. Insufficient manpower, inadequate facility and equipment, and long-term stress of caring for the critically ill post huge challenges to the nurses. There is common concern about nurses' own mental health. Burnout is commonly reported by nurses.

On the positive side, the pandemic has made the professional values held by nurses more prominent. During the long and painful battle against the pandemic, the nurses themselves, recipients of care, fellow members of the healthcare team as well the whole society have recognised how well nurses demonstrated these values (Chan, 2020). Despite the high risk of catching the fatal COVID infection, nurses are committed to alleviating suffering, supporting life, and helping both the patient and significant others. In the case of the individual dying from COVID, the nurse often becomes the patient's next of kin while the significant others are not allowed to stay close to the dying person. Many nurses have been playing such a role with complex reactions and heavy emotional load, on one hand protecting the relatives of the dying, on the other offering the best care for the dying. Without similar experiences, public are often amazed how nurses cope with such extreme pressure. Nurses deserve the highest regards by all members in the society. They should give themselves a big round of applause.

Apart from personal commitment and acknowledgement by the public, nurses need much support to keep them going. Resources like personal protection equipment, reasonable workload arrangement, measures to support the nurses' family and updated information on COVID development are essential. Frequent feedback on the use of equipment and facilities should help timely supplementation of support for nurses (Ke, Chan, Kong et al., 2021). A continuing assessment of the health status of nurses is helpful to ensure work and personal issues could be detected early and managed accordingly. Nurses specialized in infection control should be offering consultation to all clinical units. It is also essential to involve these frontline experts in planning and organizing the system for pandemic prevention and management, such as border control, contact tracing, quarantine organisation.

Vaccination does help! A simple message is that those vaccinated are likely to be less sick when infected with COVID, even with the new variant, Omicron. It is evident that nurses are much trusted by service users as well as the community as a whole. They are the best people to promote vaccination in the community. They can get the message across through live video and personal contacts.

During a pandemic, the nursing profession is often highlighted as a life career of choice. Similar to the case in the SARS in 2003, the number of schoolleavers opting for nursing degree increases drastically in the United Kingdom. Similar increase was observed in other places like Hong Kong. This could be inspired by the impressive performance, devotion, and sacrifices of the healthcare professionals. Nurses making their wholehearted contributions to the society should be encouraged by the choice of the younger generation.

Looking into the future, no matter how many waves of pandemic are coming, the nursing profession never fails to contribute to promoting health and saving lives of people!

Prof. Sally Wai-Chi Chan, BsC, MSc, PhD, FAAN, RN e-mail: sallychan@twc.edu.hk

\section{References}

Chan, S. (2020). Covid-19 and nursing workforce, leadership and education development - a discussion paper. Profese Online, 13(2), 30-35. https://doi.org/10.5507/pol.2020.015

Ke, Q. H., Chan, S., Kong, Y., Fu, J. G., Li, W. G., Shen, Q., \& Zhu, J. (2021). Frontline nurses' willingness to work during the COVID-19 pandemic: a mixed-methods study. Journal of Advanced Nursing, 77(9), 3880-3893. https://doi.org/10.1111/jan.14989 